\title{
Review "Results of treatment of chronic osteomyelitis by gutter procedure and muscle flap transposition operation"
}

\author{
Yueju Liu $\cdot$ Han Li $\cdot$ Yingze Zhang • \\ Yang Luo
}

Received: 23 April 2014/ Accepted: 30 June 2014/Published online: 19 July 2014

(C) Springer-Verlag France 2014

\section{To the Editor}

Gokalp et al. [1] have done an excellent study on the treatment of chronic osteomyelitis by gutter procedure and muscle flap transposition operation, which may be a successful mode of therapy when performed correctly and supported by long-term antibio-therapy. However, we find an obvious mistake in Gokalp MA's study. The author said that "Written informed consents were obtained from the participants"; however, there is a 2-year-old child in this study, how should he can finished his written informed consents? Therefore, we advise this sentence was revised to "Written informed consents were obtained from the participants and their guardians."

Through our limited experience, the chronic osteomyelitis of children $<3$ years was very difficult to deal with, which is especially hard to choose appropriate antibiotic. However, the author even used washing drainage daily with $1,000 \mathrm{ml}$ physiological saline including $80 \mathrm{mg}$ gentamicin for 2 days. As we know, the gentamicin has highly

Y. Liu $(\bowtie) \cdot$ H. Li $\cdot$ Y. Zhang · Y. Luo

Department of Traumatic Emergency Center, Third Hospital of

Hebei Medical University, No. 139 Zi Qiang Road,

Shijiazhuang 050051, Hebei, People's Republic of China

e-mail: liuyueju1983@gmail.com

H. Li

e-mail: 981847462@qq.com

Y. Zhang

e-mail: yzlingliu@126.com

Y. Luo

e-mail: 951256921@qq.com

Y. Liu · H. Li · Y. Zhang · Y. Luo

Key Orthopaedic Biomechanics Laboratory of Hebei Province,

Shijiazhuang, People's Republic of China ototoxicity and nephrotoxicity, which is prohibited for young children. So we wonder how it can be used in this study. And we do not know which long bone of this child suffering chronic osteomyelitis, but we know even the femoral shaft of a only 2-year-old children is very small, with a diameter of approximately $1.5 \mathrm{~cm}$. But the width of the area was determined to be smaller than $1 / 6$ of the bony circumference in order not to form a fracture in this article, which is approximately $3 \mathrm{~mm}$. So we want to know how the holes were punched on this marked area at 5-mm intervals using a drill in order to form a 3-mm gutter? It is so difficult a thing that we have not finished it till now in a 2-year-old child.

Conflict of interest The authors confirm that there are no known conflicts of interest associated with this publication and that there has been no significant financial support for this work that could have influenced its outcome.

\section{Reference}

1. Gokalp MA, Guner S, Ceylan MF et al (2014) Results of treatment of chronic osteomyelitis by "gutter procedure and muscle flap transposition operation". Eur J Orthop Surg Traumatol 24:415-419 